Submitted to The Astrophysical Journal Letters

\title{
The ACS Survey of Galactic Globular Clusters! M54 and Young Populations in the Sagittarius Dwarf Spheroidal Galaxy
}

\author{
Michael H. Siegel ${ }^{2}$, Aaron Dotter ${ }^{3}$, Steven R. Majewski ${ }^{4}$, Ata Sarajedini ${ }^{5}$, Brian Chaboyer $^{3}$, \\ David L. Nidever ${ }^{4}$, Jay Anderson ${ }^{6}$, Antonio Marín-Franch ${ }^{5,7}$, Alfred Rosenberg ${ }^{7}$, Luigi R. \\ Bedin $^{8}$, Antonio Aparicio ${ }^{7}$, Ivan King ${ }^{9}$, Giampaolo Piotto ${ }^{10}$, I. Neill Reid ${ }^{8}$
}

\begin{abstract}
We present new Hubble Space Telescope photometry of the massive globular cluster M54 (NGC 6715) and the superposed core of the tidally disrupted Sagittarius (Sgr) dSph galaxy as part of the ACS Survey of Galactic Globular Clusters.
\end{abstract}

\footnotetext{
${ }^{1}$ The fourth paper in the series based on observations with the NASA/ESA Hubble Space Telescope, obtained at the Space Telescope Science Institute, which is operated by AURA, Inc., under NASA contract NAS 5-26555, under programs GO-10775 (PI: Sarajedini).

${ }^{2}$ University of Texas, McDonald Observatory, 1 University Station, C1402, Austin TX, 78712 (siegel@astro.as.utexas.edu)

${ }^{3}$ Department of Physics and Astronomy, Dartmouth College, 6127 Wilder Laboratory, Hanover, NH 03755

(Aaron.L.Dotter@dartmouth.edu,chaboyer@heather.dartmouth.edu)

${ }^{4}$ Dept. of Astronomy, University of Virginia, P.O. Box 400325, Charlottesville, VA 22904-4325 (srm4n@virginia.edu, dln5q@virginia.edu)

${ }^{5}$ Department of Astronomy, University of Florida, 211 Bryant Space Science Center, Gainesville, FL 32611 (ata@astro.ufl.edu, amarin@astro.ufl.edu)

${ }^{6}$ Department of Physics and Astronomy, Rice University MS-108, Houston, TX 77005 (jay@eeyore.rice.edu)

${ }^{7}$ Instituto de Astrofísica de Canarias, Vía Láctea s/n, E-38200 La Laguna, Spain (alf@iac.es,antapaj@iac.es,amarin@iac.es)

${ }^{8}$ Space Telescope Science Institute, 3700 San Martin Drive, Baltimore MD 21218 (bedin@stsci.edu, inr@stsci.edu)

${ }^{9}$ Dept. of Astronomy, Univ. of Washington, Box 351580, Seattle, WA 98195-1580 (king@astro.washington.edu)

${ }^{10}$ Dipartimento di Astronomia, Università di Padova, 35122 Padova, Italy (piotto@pd.astro.it)
} 
Our deep ( $F 606 W \sim 26.5)$, high-precision photometry yields an unprecedentedly detailed color-magnitude diagram showing the extended blue horizontal branch and multiple main sequences of the M54+Sgr system. The distance and reddening to M54 are revised using both isochrone and main-sequence fitting to $(m-M)_{0}=17.27$ and $E(B-V)=0.15$. Preliminary assessment finds the M54+Sgr field to be dominated by the old metal-poor populations of Sgr and the globular cluster. Multiple turnoffs indicate the presence of at least two intermediate-aged star formation epochs with 4 and $6 \mathrm{Gyr}$ ages and $[\mathrm{Fe} / \mathrm{H}]=-0.4$ to -0.6 . We also clearly show, for the first time, a prominent, $\sim 2.3$ Gyr old Sgr population of nearsolar abundance. A trace population of even younger ( 0.1-0.8 Gyr old), more metal-rich $([\mathrm{Fe} / \mathrm{H}] \sim 0.6)$ stars is also indicated. The Sgr age-metallicity relation is consistent with a closed-box model and multiple (4-5) star formation bursts over the entire life of the satellite, including the time since Sgr began disrupting.

Subject headings: globular clusters: individual (M54); galaxies: individual (Sagittarius); galaxies: star clusters; galaxies: stellar content

\section{Introduction}

M54 (NGC 6715) is the second most massive Galactic globular cluster and, at first blush, a canonical "old halo" cluster: ancient, metal poor and with a very extended blue horizontal branch (HB; Harris 1996). However, M54 has been shown (Ibata et al. 1994; Sarajedini \& Layden 1995, hereafter "SL95"; Majewski et al. 2003; Monaco et al. 2005a; Siegel et al. 2007) to lie at the photometric center and distance of the Sagittarius (Sgr) dwarf spheroidal (dSph) galaxy, which is merging with the Milky Way (Ibata et al. 1994), with tidal arms encircling the Galaxy (e.g., Ibata et al. 2001b, Newberg et al. 2002; Majewski et al. 2003). This has prompted discussion of whether M54 may be the nucleus of the Sgr dSph (e.g., SL95; DaCosta \& Armandroff 1995; Bassino \& Muzzio 1995; Layden \& Sarajedini 2000, hereafter "LS00") around which later star formation occurred. However, based on the existence of two distinct ancient, metal-poor populations (MPPs) with different radial profiles (SL95), and the observation that Sgr would be nucleated even if M54 were ignored (Monaco et al. 2005a), it seems likely that M54 formed separately from Sgr and was pulled into the dSph center through dynamical friction.

Photometric studies of M54 provide information on both the cluster and the Sgr core (SL95, Layden \& Sarajedini 1997; LS00), and, in combination with spectroscopic efforts, have confirmed the metal-poverty and ancient age of both the cluster $([\mathrm{Fe} / \mathrm{H}]=-1.5$ to -1.8 , 14-15 Gyr; Brown et al. 1999, LS00) and Sgr's distinct MPP $([\mathrm{Fe} / \mathrm{H}]=-1.3$, age $=10-11$ 
Gyr, LS00). Large surveys of Sgr's core are dominated by intermediate stellar populations $([\mathrm{Fe} / \mathrm{H}]=-0.4$ to -0.7 , 5-8 Gyr; SL95, LS00, Bellazzini et al. 2006a; hereafter B06), though this is likely due to Sgr's MPPs having been selectively stripped into tidal tails (Chou et al. 2007). There have also been hints of a young metal-rich population (2.5 Gyr, -0.4 dex; SL95, LS00), including stars of solar-abundance (Smecker-Hane \& McWilliam 2002; Monaco et al. 2005b, Chou et al. 2007). When the properties of stellar populations in and around M54 are combined with Sgr clusters near the core (Terzan 7, Terzan 8, Arp 2; Ibata et al. 1994) or in the Sgr tidal stream (Pal 12, Dinescu et al. 2000; Pal 2, Majewski et al. 2004; Whiting 1, Carraro et al. 2006), Sgr has an age-metallicity relation (AMR) consistent with a simple closed-box model (see Fig. 18 of LS00).

Because Sgr has been disrupting for at least 2.5-3.0 Gyr (Law et al. 2005), and likely longer, it is a unique laboratory for exploring star formation in the context of hierarchical galaxy formation. Not only can we potentially connect the star formation and enrichment history of a specific satellite galaxy with relevant timescales and events in its interaction, but also ascertain in detail the populations this disintegrating system has donated and is still donating to the Galaxy. We contribute to this effort by clarifying the Sgr+M54 stellar populations with new $H S T / A C S / W F C$ photometry from the ACS Survey of Galactic Globular Clusters (Sarajedini et al. 2007, hereafter Paper I).

\section{Observations and Data Reduction}

The ACS survey observed 65 globulars in the $F 606 W(\sim V)$ and $F 814 W(\sim I)$ filters with HST/ACS/WFC. PSF photometry (Anderson et al., 2007, in prep) is Vega-calibrated using the charge-transfer efficiency corrections of Reiss \& Mack (2004), calibration procedures in Bedin et al. (2005) and zero points of Sirianni et al. (2005). This is supplemented by photometry of isolated saturated stars from short exposures salvaged by summing all associated charge — a procedure previously applied by Gilliland (2004). For M54, the observation and reduction pipeline produces twelve magnitudes of precise photometry from nearly the tip of the red giant branch (RGB) to several magnitudes below the main sequence turnoff (MSTO). Our extraction of nearly 390,000 detections in the ACS/WFC field leaves a star-subtracted image nearly devoid of flux. However, many of the detections do not provide precise photometry due to source faintness, charge bleeding, cosmic rays and close neighbors. For this initial examination of the color-magnitude diagram (CMD) we used the trend of quality-of-fit against magnitude to select 60,000 sources with the most star-like profiles and $<10 \%$ of the flux in their PSF aperture from other stars. 
Fig. 1.- ACS photometry of the M54 field. Panel (a) shows the CMD of 60,000 stars selected to be PSF-like and to have less than $10 \%$ contribution of neighbor stars to their integrated light. Panel (b) shows a Hess diagram of the field with an overlayed schematic describing the various populations in the M54+Sgr field. The dotted line is the Sgr MPP as defined in LS00. Panel (d) shows the Hess diagram overlayed with theoretical isochrones describing the inferred stellar populations while Panel (c) shows the simulated CMD described in the text.

\section{Color-Magnitude Diagram Features}

The CMD of the M54 field (Fig. 1a) shows an extraordinary array of features: an extended blue and red HB, at least two red RGBs, multiple MSTOs and multiple MSs. Fig. $1 \mathrm{~b}$ shows a Hess diagram overlayed with a schematic description of the features seen in the complex M54+Sgr CMD, which we now detail:

The Old M54 Population: The most prominent feature in Fig. 1a is the strong MS and RGB from the combined M54 and Sgr MPPs (shown to have slightly different RGBs by SL95 and LS00). The red line in Fig. 1b is a fiducial through the MS and RGB centers for the MPPs determined using techniques described in Rosenberg et al. (2006) that interactively fit Gaussians to the top $20 \%$ of the magnitude-color distribution orthogonal to the cluster sequence in $0.4 \mathrm{mag}$ wide overlapping bins stepped every 0.04 mag between $F 814 W=15$ and 25.1 The Gaussian widths provide weights for $\chi^{2}$ fits of main-sequence fiducials and isochrones. The MPPs are also reflected in the asymptotic giant branch and lengthy HB running from a prominent red HB through the RR Lyrae gap to an extended blue HB. The latter also includes the "blue hook" population of extremely hot HB stars identified by Rosenberg et al. (2004). The extreme HB stars are centrally concentrated in the field, consistent with membership in the old, metal-poor cluster. Similar "blue hook" stars have also been identified in the massive clusters $\omega$ Centauri and NGC 2808 (Moehler et al. 2002, 2004).

The narrowness of the MPP sequence allows a straight-forward measurement of the distance and reddening to M54 using MS and isochrone fitting. To minimize any confusing effect of Sgr's old MS, we fit distance and reddening both by eye and with a $\chi^{2}$ minimization routine to the fiducial line shown in Fig. 1b, which is defined by the dominant M54 MS and RGB. Metal-poor isochrones were taken from Dotter et al. (2007, Paper II) with

\footnotetext{
${ }^{1}$ The metal-poor SGB shows evidence of bifurcation from the overlapping MPPs of M54 and Sgr. However, our Gaussian fits follow the center of the dominant M54 sequence.
} 
$[\alpha / \mathrm{Fe}]=+0.2$ in agreement with Brown et al. (1999) and $[\mathrm{Fe} / \mathrm{H}]$ allowed to vary from -2 to -1.5 (in 0.1 dex steps). The optimal fit uses an isochrone of $13 \mathrm{Gyr}$ with $[\mathrm{Fe} / \mathrm{H}]=-1.8$ at $(m-M)_{0}=17.23$ and $E(B-V)=0.17$. The Hess diagram (Fig. 1d) indicates good agreement between the theoretical isochrone (red line) and the observed MPP sequence. Empirical MSfitting used the fiducials defined in Paper I for the NGC 6752 and M92 clusters weighted heaviest near $M_{F 606 W} \sim+4.0$. Interpolating between the two clusters to the isochrone abundance of M54 $([\mathrm{Fe} / \mathrm{H}]=-1.8)$ yields $E(B-V)=0.14$ and $(m-M)_{0}=17.31$. The mean of the MS and isochrone measures - $(m-M)_{0}=17.27, E(B-V)=0.152$ - is in reasonable agreement with the RGB tip distance of Monaco et al. (2004) and the RR Lyrae distance of LS00.

Our subsequent analysis assumes that M54 and Sgr have the same reddening and distance because M54 appears to lie at the center of $\operatorname{Sgr}(\S 1)$ and the distance uncertainty $(\sim 0.05 \mathrm{mag}$ or $0.6 \mathrm{kpc})$ is large enough to mask any small discrepancy. A small difference in distance modulus could slightly alter the inferred ages of the Sgr populations, as shown in B06.

The Intermediate Population(s): The intermediate Sgr population ("SInt"), described in detail by LS00 and B06 (as "Population A"), dominates wide-field surveys of Sgr. The SInt features in Fig. 1b (orange) include a prominent red clump, a redder RGB and a redder MS that begins to diverge from the MPP MS below $F 606 W=24$. Monaco et al. (2005b) and Sbordone et al. (2006) determined the abundances of the primary intermediate Sgr population to be $[\mathrm{Fe} / \mathrm{H}]=-0.4$ and $[\alpha / \mathrm{Fe}]=-0.2$. While B06 suggest an age for this population of $8 \pm 1.5 \mathrm{Gyr}$, at $(m-M)_{0}=17.2$ their SInt age is 5-6 Gyr. Isochrones corresponding to the measured abundances and younger B06 age match the SInt RGB and the bluer MSTO emerging just beyond the MPP MSTO. However, the CMD appears to have a broad (or perhaps two distinct) MSTOs between the old and "young" MSTOs, which indicates multiple bursts. $[\mathrm{Fe} / \mathrm{H}]=-0.6,6 \mathrm{Gyr}$ and $[\mathrm{Fe} / \mathrm{H}]=-0.5,4.5$ Gyr isochrones (orange lines, Fig. 1d) seem to best reproduce the most apparent intermediate MSTO features.

The Young Population(s): The bluest strong MSTO ("SYng", green line in Fig. 1b) corresponds to a significantly younger population than SInt. Though hinted at before (Mateo et al. 1995; Bellazzini et al. 1999a,b; LS97; LS00), only weak constraints could be applied to what was previously an indistinct CMD feature. The superior $A C S$ photometry of the M54+Sgr core, however, clearly reveals this as a young metal-rich MSTO with a convective hook at $F 606 W=19.5$. The best fit to SYng is a $2.3 \mathrm{Gyr}$ isochrone with $[\mathrm{Fe} / \mathrm{H}]=-0.1$ and

\footnotetext{
${ }^{2}$ We checked the data for indications of differential reddening using methods outlined in Paper I. The results indicate minimal differential reddening.
} 
$[\alpha / \mathrm{Fe}]=-0.2$ (green line, Fig. 1d) 3 similar to, but more metal-rich than, the youngest Sgr population described in LS00. SYng is younger than the minimum interval over which Sgr has been disrupting ( 2.5-3 Gyr ago, Law et al. 2005).

Finally, a sparse, bright MS can be seen above the other MSs in the CMD and extending blueward as a "spray" of stars extending above the 2.3 Gyr MSTO through the blue HB (blue features in Fig. 1b). While the latter could be blue straggler stars, a very young, metal-rich population ("SVYng") is also hinted at by the clump of stars below the Sgr red clump which are too blue to be a metal-rich or intermediate RGB bump but faint enough to be a young red HB clump. The lack of a distinct MSTO or RGB associated with SVYng and the potential confusing contribution of binaries and blue stragglers makes further statements regarding its age/composition speculative. However, the SVYng stars could represent the youngest, most metal rich M54+Sgr population. The bright MS extends beyond the sample isochrones at $[\mathrm{Fe} / \mathrm{H}]=+0.56,100$ and $800 \mathrm{Myr}$ (blue lines) overlayed in Fig. 1d, suggesting protracted, recent star formation in Sgr.

\section{The Star Formation History of the M54 Field}

Untangling the multiple stellar populations of Sgr, including the contribution of binaries, can be aided by population synthesis. Using the isochrones fit in $\S 3$, we reconstructed the star formation history (SFH) of the M54 field using the StarFISH population synthesis code (Harris \& Zaritsky 2001). StarFISH uses a set of isochrones, an error model (in this case, an analytical one) and fixed $(m-M), E(B-V)$ and IMF to construct a library of CMD probability functions. It then iteratively finds which combination of synthetic CMDs reproduces the observed CMD, varying the amplitude of the input populations by downhill simplex until convergence. The result is an age-metallicity-amplitude SFH of the field.

We set the distance and reddening to the values derived in $\S 3$ and used a Salpeter IMF. After some initial variation, we fixed the well-defined MPP and SYng populations while allowing SVYng to vary in age from 0.1 to 0.9 Gyr. The SInt populations were allowed to initially vary from $[\mathrm{Fe} / \mathrm{H}]=-0.3,[\alpha / \mathrm{Fe}]=-0.2$ to $[\mathrm{Fe} / \mathrm{H}]=-1.5,[\alpha / \mathrm{Fe}]=+0.2$ and over $2-15$ Gyr. This window was gradually narrowed and a final fit was composed by hand to provide better age definition and reproduction of the MSTO region

\footnotetext{
${ }^{3}$ A satisfactory fit can also be obtained with a 1.75 Gyr, solar abundance isochrone with $Y=0.33$. At this time, however, we have no reason to suspect an enhanced He abundance in Sgr.
} 
The derived M54+Sgr SFH (Fig. 2)4 is dominated by the M54 MPP, which contributes $\sim 75 \%$ of the simulated stars. Sgr contributes a small MPP and a broad range of SInt stars. SYng is strong and distinct while SVYng is weak and tenuous in the CMD (Fig. 1c). The populations follow a closed-box AMR model similar to that of LS00 (dotted line) but with faster enrichment (solid line).

We supplemented the StarFISH-simulated MSs and RGBs with synthetic HBs constructed with the He-burning tracks and modeling code from Paper II. Mass distributions were constructed with an upper limit supplied by the fitted isochrones with an average mass loss of $0.1 M_{\odot}$ for the M54 MPP, $0.05 M_{\odot}$ for the Sgr MPP and SInt, and no mass loss for SYng. The amount of mass loss for each population was set to best reproduce the observed HB. All models used a mass loss dispersion of $0.05 M_{\odot}$. The number of HB stars for each population was set by the appropriate R-ratio given the assumed He abundance.

Fig. 1c shows the simulated CMD. Our relatively simple simulations recreate the salient features of the M54+Sgr field, including the broad MS, the complex MSTO, the bifurcated SGB5, the doubled RGB and the long blue HB. The simulated HB has a steeper slope than the real HB, which could be corrected if the M54 abundance were raised by a few 0.1 dex.

While SYng and SVYng are stronger than the SInt population(s) in our ACS field, this does not apply to Sgr over larger scales, where other studies (e.g., LS00 and B06) show SInt dominating. Surveys of Sgr's tidal arms have shown that the stars it is contributing to the halo are, on average, even more metal-poor (Bellazzini et al. 2006b; Chou et al. 2007). Our analysis reveals the presence of recently formed stars in the center of Sgr, further affirming the strong metallicity gradient in the system.

\section{REFERENCES}

Bassino, L. P., \& Muzzio, J. C. 1995, The Observatory, 115, 256

Bedin, L., et al. 2005, MNRAS, 357, 1038

Bellazzini, M., Ferraro, F. R., \& Buonanno, R., 1999a,MNRAS, 304, 633

\footnotetext{
${ }^{4}$ Isochrones of similar age-abundance combinations create degenerate solutions in StarFISH. This is accounted for by "locking" together degenerate groups of isochrones into single CMD probability functions. The points in Figure 2 are Gaussians set to the center and range of each locked isochrone group.

${ }^{5}$ Our analysis of NGC 1851 (Piotto et al. 2007) also shows a bifurcated SGB, which we ascribe to a 1 Gyr age spread.
} 
Bellazzini, M., Ferraro, F. R., \& Buonanno, R., 1999b,MNRAS, 307, 619

Bellazzini, M., Correnti, M., Ferraro, F. R., Monaco, L., \& Montegriffo, P. 2006a, A\&A, 446, 1 [B06]

Bellazzini, M., Newberg, H. J., Correnti, M., Ferraro, F. R. \& Monaco, L., 2006b, A\&A, 457,21

Brown, J. A., Wallerstein, G., \& Gonzalez, G. 1999, AJ, 118, 1245

Carraro, G., Zinn, R. \& Moni Bidin, C., 2006, A\&A, 466, 181

Chou, M., et al. 2007, ApJ, submitted, astro-ph/0605101

Da Costa, G. S., \& Armandroff, T. E. 1995, AJ, 109, 2533

Dinescu, D. I., Majewski, S. R., Girard, T. M., \& Cudworth, K. M. 2000, AJ, 120, 1892

Dotter, A., Chaboyer, B., Jevremović, D., Baron, E., Ferguson, J. W., Sarajedini, A., Anderson, J. 2007, AJ, 134, 376

Gilliland, R. L. 2004, STScI Inst. Sci. Rep. ACS 2004-001 (Baltimore: STScI)

Harris, J. \& Zaritsky, D.,2001, ApJ, 136, 25

Harris, W. E., 1996, AJ, 112, 1487

Ibata, R. A., Gilmore, G., \& Irwin, M. J. 1994, Nature, 370, 194

Ibata, R., Lewis, G. F., Irwin, M., Totten, E., \& Quinn, T. 2001, ApJ, 551, 294

Law, D. R., Johnston, K. V., \& Majewski, S. R. 2005, ApJ, 619, 807

Layden, A. C., \& Sarajedini, A. 1997, ApJ, 486, L107

Layden, A. C., \& Sarajedini, A. 2000, AJ, 119, 1760 [LS00]

Majewski, S. R., Skrutskie, M. F., Weinberg, M. D., \& Ostheimer, J. C. 2003, ApJ, 599, 1082

Majewski, S. R., et al. 2004, AJ, 128, 245

Mateo, M., Udalski, A., Szymanski, M., Kaluzny, J., Kubiak, M., \& Krzeminski, W., 1995a, AJ, 109, 588

Moehler, S., Sweigart, A. V., Landsman, W. B., \& Dreizler, S. 2002, A\&A, 395, 37 
Moehler, S., Sweigart, A. V., Landsman, W. B., Hammer, N. J., \& Dreizler, S. 2004, A\&A, 415,313

Monaco, L., Bellazzini, M., Ferraro, F. R. \& Pancino, E., 2004, MNRAS, 353, 874

Monaco, L., Bellazzini, M., Ferraro, F. R. \& Pancino, E., 2005a, MNRAS, 356, 1396

Monaco, L., Bonifacio, P., Ferraro, F. R., Marconi, G., Pancino, E., Sbordone, L, \& Zaggia, S. 2005b, A\&A, 441, 141

Piotto, G., et al. 2007, ApJ, submitted

Reiss, A., \& Mack, J. 2004, STScI Inst. Sci. Rep. ACS 2004-006 (Baltimore: STScI)

Rosenberg, A., Recio-Blanco, A., \& García-Marín, M. 2004, ApJ, 603, 135

Rosenberg, A., Marin-Franch, A., Aparicio, A., Piotto, G., Chaboyer, B. \& Sarajedini, A., 2006, BAAS, 209, 100.13

Sarajedini, A., \& Layden, A. C. 1995, AJ, 109, 1086

Sarajedini, A. et al., 2007, AJ, 133, 1658 (Paper I)

Siegel, M., Majewski, S. R., Sarajedini, A., Chaboyer, B. \& Rosenberg, A., 2006, BAAS, 209, 100.12

Sirianni, M. et al. 2005, PASP, 117, 1049

Smecker-Hane, T. \& McWilliam, A., 2002, AJ, submitted, astro-ph/0205411

Sollima, A., Ferraro, F. R., Bellazzini, M., Origlia, L., Straniero, O., \& Pancino, E. 2007, ApJ, 654, 915 


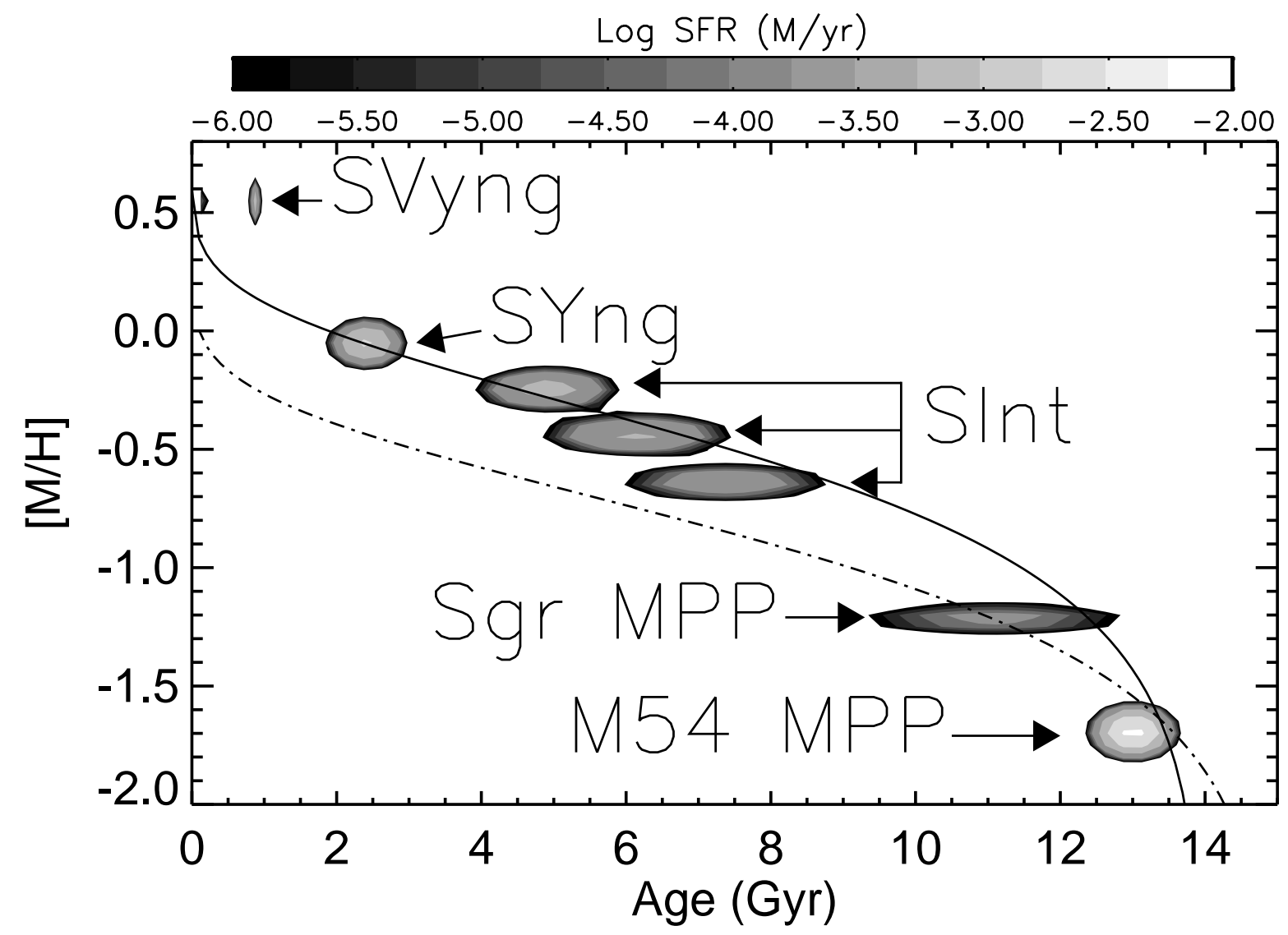

Fig. 2.- The simulated SFH of the M54 field. Distinct contributions are from the metalpoor M54 population (M54 MPP), Sgr's metal-poor (Sgr MPP), and Sgr's young (SYng) populations The intermediate Sgr population (SInt) is broad and composed of multiple bursts or continuous star formation. There appears to be some contribution from a very young Sgr population (SVYng). The dotted line is the AMR from LS00 using a simple closed-box model; the solid line an updated model with faster enrichment. 
This figure "m54_cmd.jpg" is available in "jpg" format from: http://arxiv.org/ps/0708.0027v1 